\title{
Effect of Increasing the Visitation Time on Pain Parameters and Consciousness Level of Intensive Care Unit's Patients
}

\author{
Asgari P. ${ }^{1}$ MSc, Mahmoudi M.* ${ }^{*} S c$, Hekmatpou D. ${ }^{1}$ PhD, Khajehgoodary M. $^{1}$ MSc, Rafiei F. ${ }^{2}$ MSc
}

*Medical Surgical Nursing Department, Nursing \& Midwifery Faculty, Arak University of Medical Sciences, Arak, Iran

${ }^{1}$ Medical Surgical Nursing Department, Nursing \& Midwifery Faculty, Arak University of Medical Sciences, Arak, Iran

${ }^{2}$ Statistics Department, Nursing \& Midwifery Faculty, Arak University of Medical Sciences, Arak, Iran

\begin{abstract}
Aims: One of the most important needs of hospitalized patients in intensive care units is patients visiting by their family members, but it is very limited in these units. The aim of this study was to determine the effect of increased visiting to patients' time on pain and level of consciousness of hospitalized patients in intensive care units.

Materials \& Methods: In this clinical trial, 60 hospitalized patients in intensive care units of Vali-Asr Hospital in Arak City, Iran, in 2013-2014 were selected by convenience sampling method and were randomly assigned into intervention and control groups. Each visiting was done for 10 minutes, 3 times a day (9and $12 \mathrm{am}$ and $15 \mathrm{pm}$ ) in the intervention group and for 10 minutes once a day $(15 \mathrm{pm})$ in the control group. Then patients' pain and level of consciousness was measured before, during, and after 10 and 30 minutes of visiting by family. Data was analyzed in SPSS 20 software using Chi-square, paired T, independent T, and repeated measure ANOVA tests.

Findings: There was no significant difference between pain and level of consciousness between 2 groups before intervention $(p>0.05)$. There was no significant difference between the means of pain and level of consciousness before and $30 \mathrm{~min}$ after the visiting at $15 \mathrm{pm}$ in control group $(\mathrm{p}>0.05)$. There were significant differences between the means of pain before and 30min after visiting at $9 \mathrm{am}, 12 \mathrm{am}$ and $15 \mathrm{pm}$ in intervention group $(\mathrm{p}<0.05)$, but no significant differences were seen in the level of consciousness ( $p>0.05$ ).

Conclusion: Increasing the time of visiting to patients decreases the pain of the hospitalized patients in intensive care units.
\end{abstract}

\section{Keywords}

Visitors to Patients [http://www.ncbi.nlm.nih.gov/mesh/68014789];

Pain [http://www.ncbi.nlm.nih.gov/mesh/68010146];

Consciousness Monitors [http://www.ncbi.nlm.nih.gov/mesh/68056805];

Intensive Care Units [http://www.ncbi.nlm.nih.gov/mesh/68007362]

\footnotetext{
${ }^{*}$ Corresponding Author

Tel: +988634173524

Fax: +988634173524

Address: Complex of Pyambar Azam, Faculty of Nursing \& Midwifery, Arak University of Medical Sciences, Sardasht Square, Arak, Iran

mokhtar.mahmoudi@arakmu.ac.ir

Received: September 15, 2015 Accepted: November 30, 2015 ePublished: December 15, 2015
} 


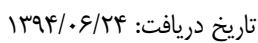

"نويسنده مسئول: mokhtar.mahmoudi@arakmu.ac.ir

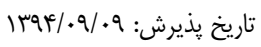

تاثير افزايش زمان ملاقات بر شاخصنهان

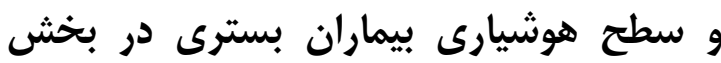

مر اقبتهاى ويزه

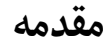

بذيرش بيمار در بخشهاى مراقبت ويزه بهدليل ترسى كه فرد در

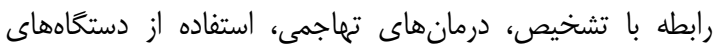

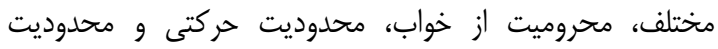

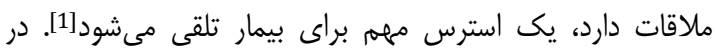

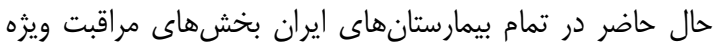

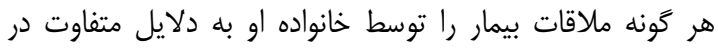

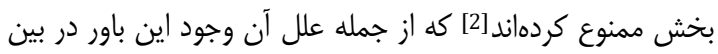

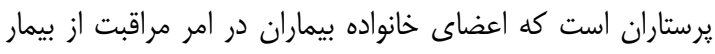

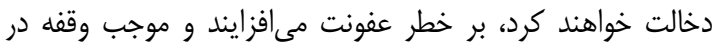

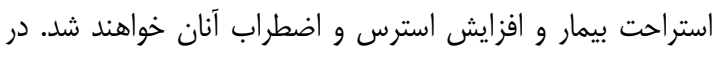

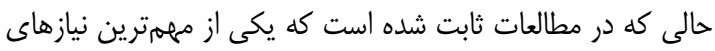

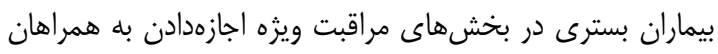

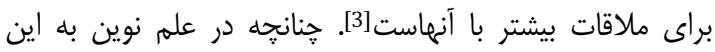

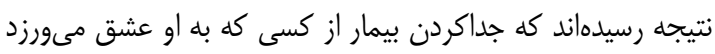

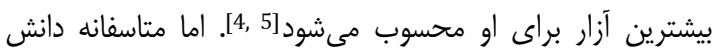

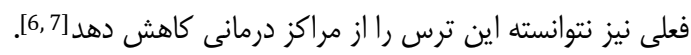

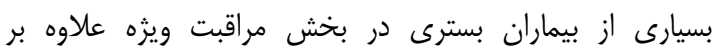

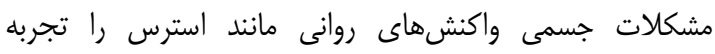

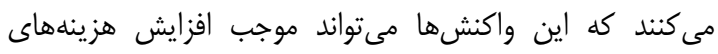

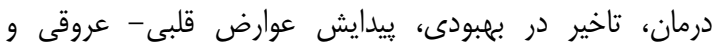

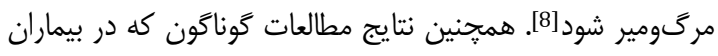

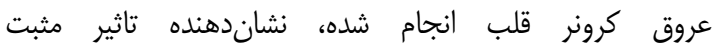

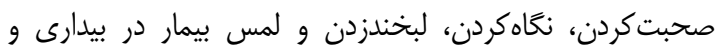

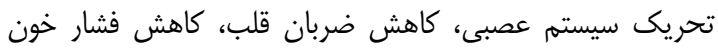

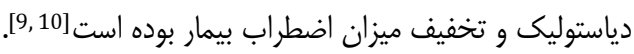

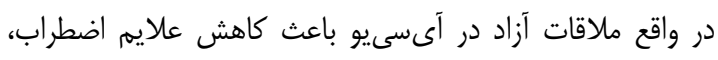

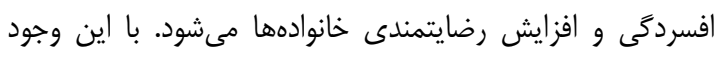

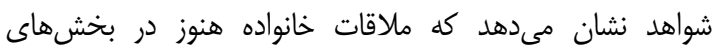

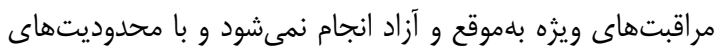

زيادى همراه است[11].

مطالعات نشان داده كه تحريكات بيمار بسترى در آى آىىيو مانند

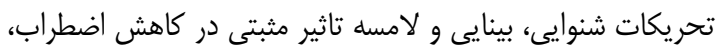

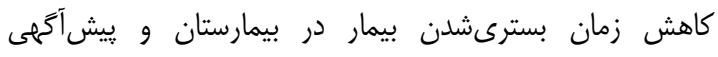

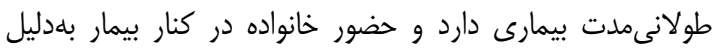

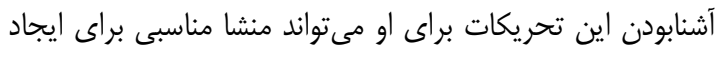

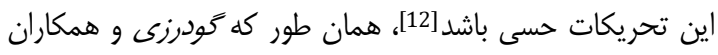

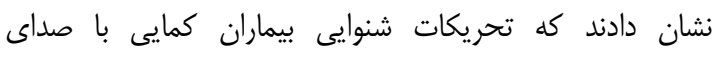

يروانه عسكَرى

كروه داخلى - جراحى، دانشكده يرستارى و مامايى، دانشكاه علوم يزشكى اراى، اراك، ايران

MSc مختار محمودى

كروه داخلى - جراحى، دانشكده يرستارى و مامايى، دانشكاه علوم يزشكى اراك، اراى، ايران

داوود حكمت يو PhD

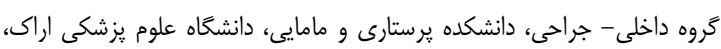
اراى، ايران

محمد خواجه تَودرى إنى

كروه داخلى - جراحى، دانشكده يرستارى و مامايى، دانشكاه علوم يزشكى اراك، اراك، ايران

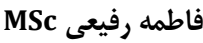

كَروه آمار، دانشكده يرستارى و مامايى، دانشكاه علوم يزشكى اراك، اراك، ايران

جكيده

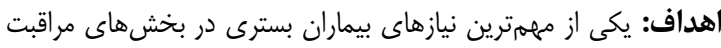

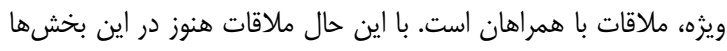

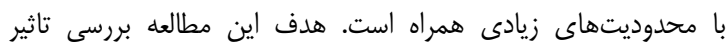

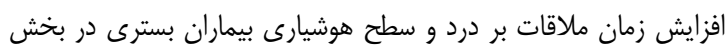

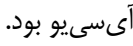

مواد و روش بودا: در اين مطالعه كارآزمايى بالينى • ع بيمار بسترى در

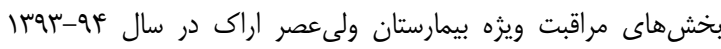

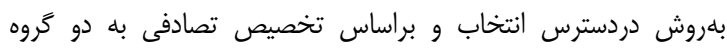

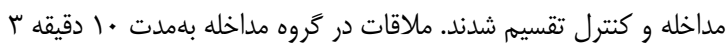

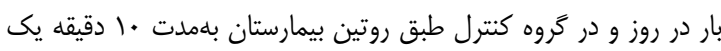

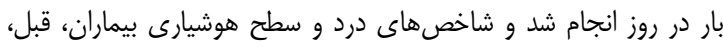

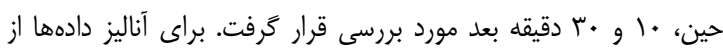

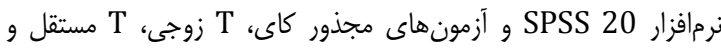
آناليز واريانس اندازههاى تكرارى استفاده شدائ

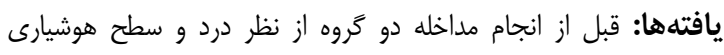

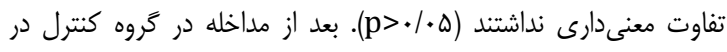

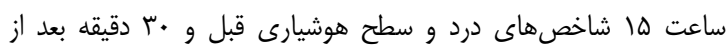

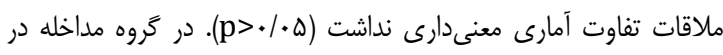

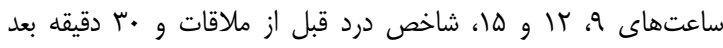

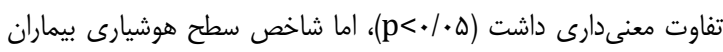

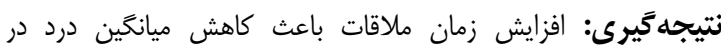

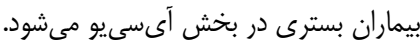

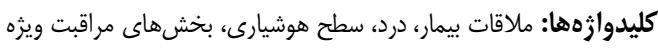

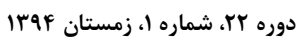

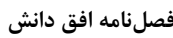

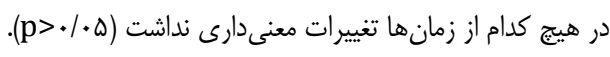




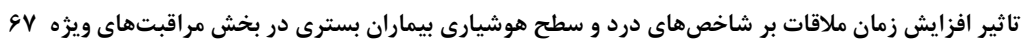

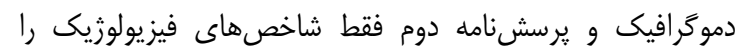

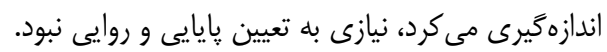

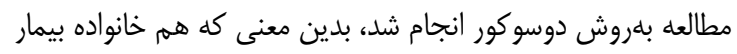

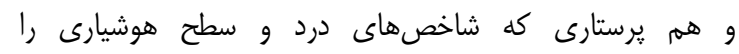

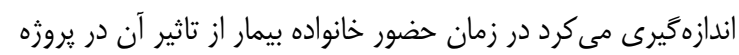
تحقيقاتى اطلاع نداشتند.

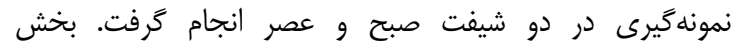

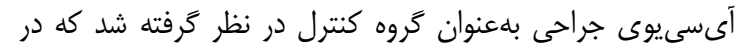

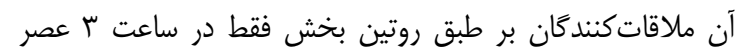

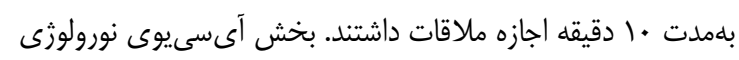

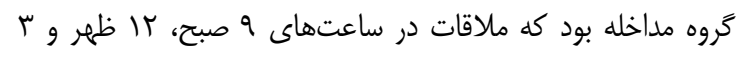

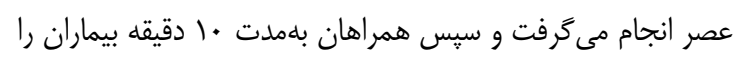

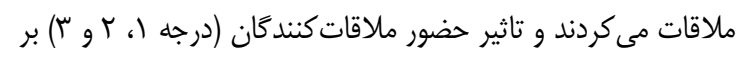

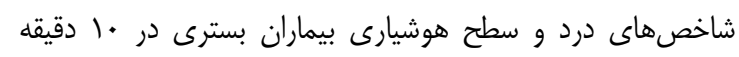

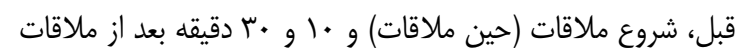

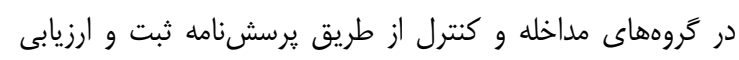

آناليز دادهها با استفاده از نرمافزار 20 SPSS و آزمونهاى آمارى

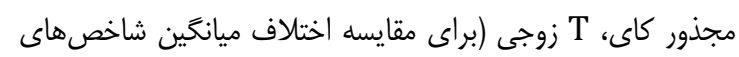

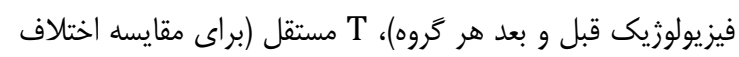

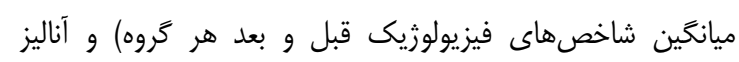

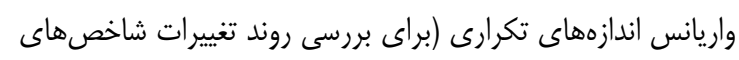
فيزيولوزيك در دو كَروه) استفاده شد.

يافتهها

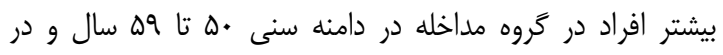

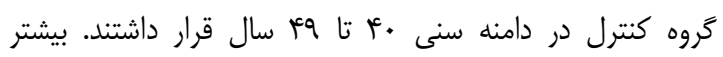

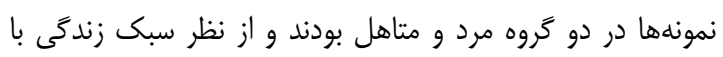

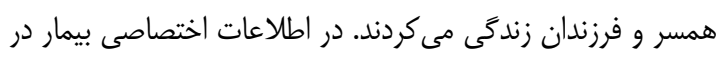

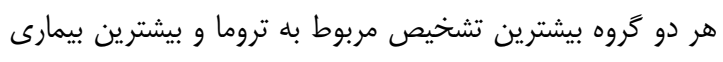

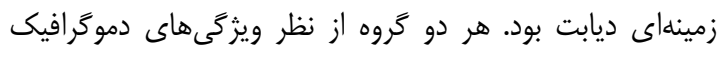

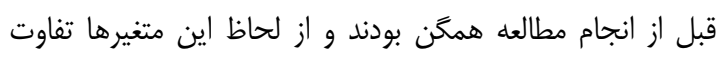

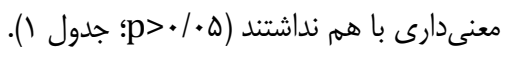

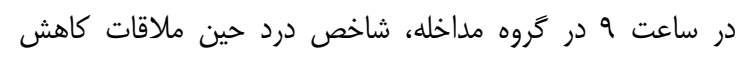

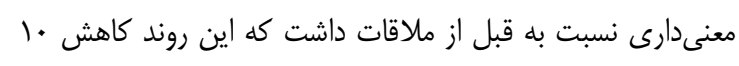

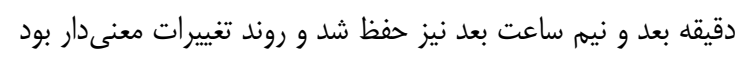

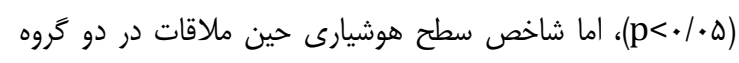

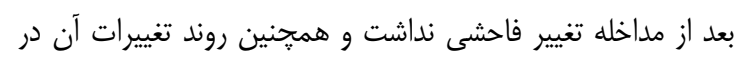

ضبطشده فرد مورد علاقه بيمار در خانواده نمره هوشيارى بيماران

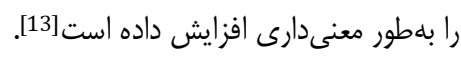

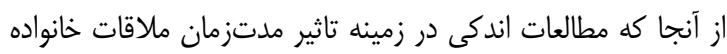

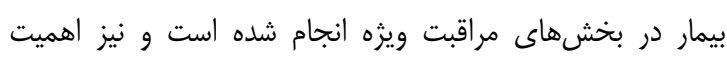

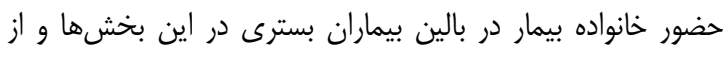

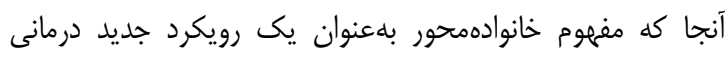

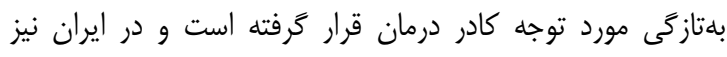

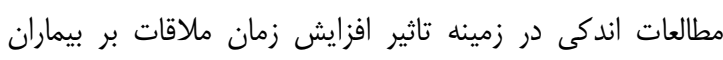

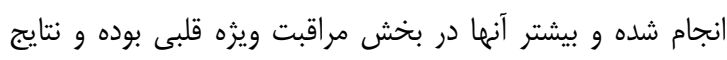

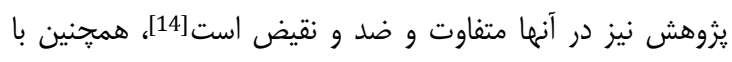

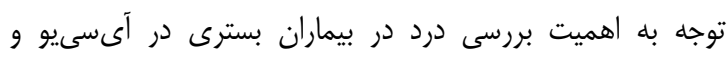

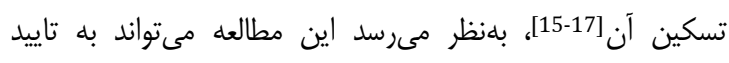

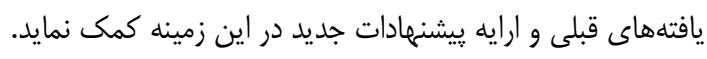

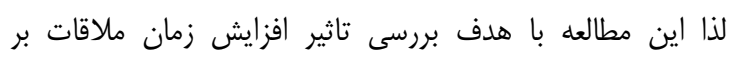

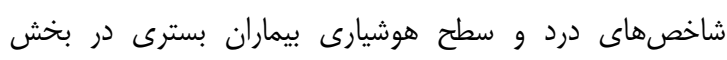
آىسى شيو انجام شد.

\section{مواد و روشها}

در اين كارآزمايى بالينى، •ع نفر از بيماران بسترى در بخشهاى

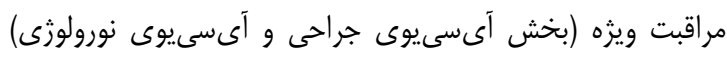

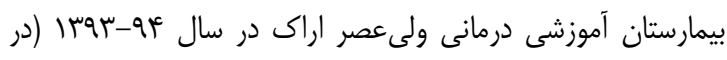

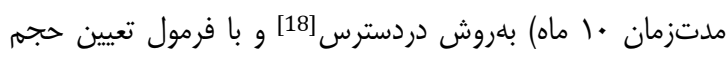

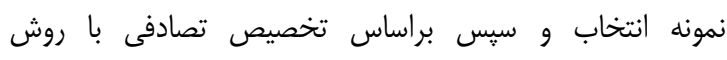

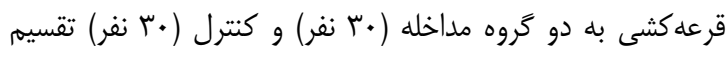

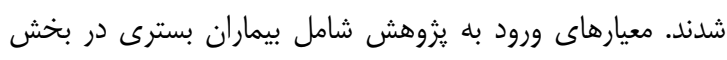

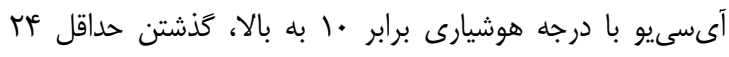

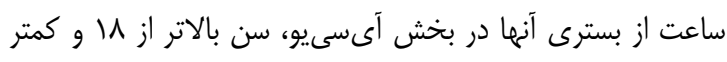

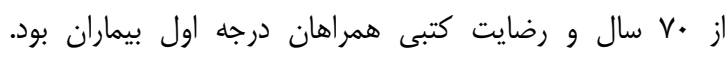

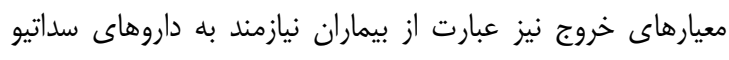

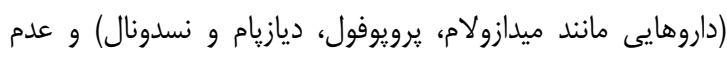

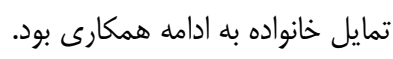

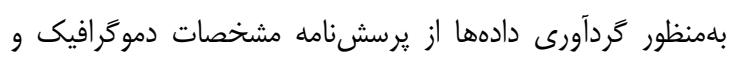

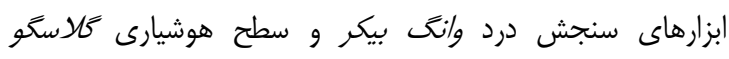

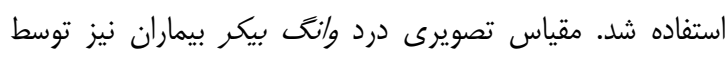

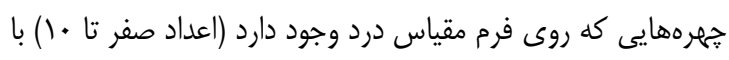

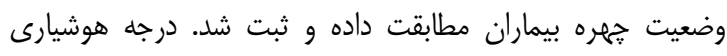

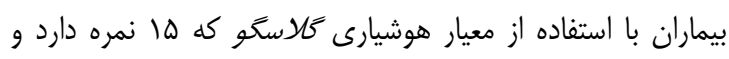

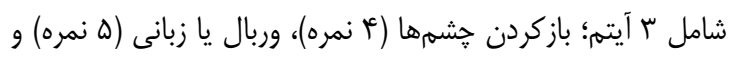

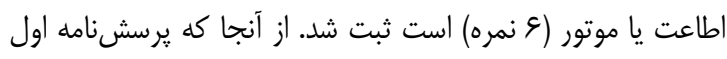




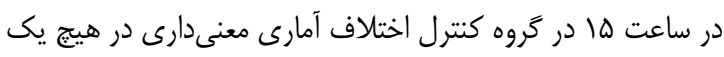

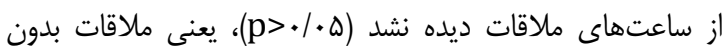

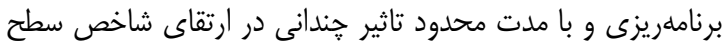

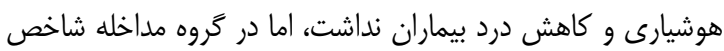

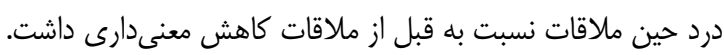

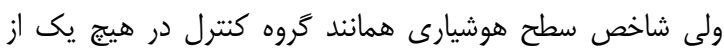

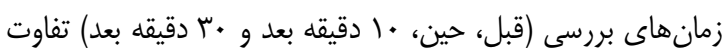

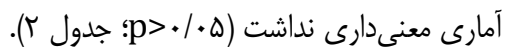

جدول r) مقايسه ميانگين شاخصهاى درد و سطح هوشيارى در گروه مداخله و

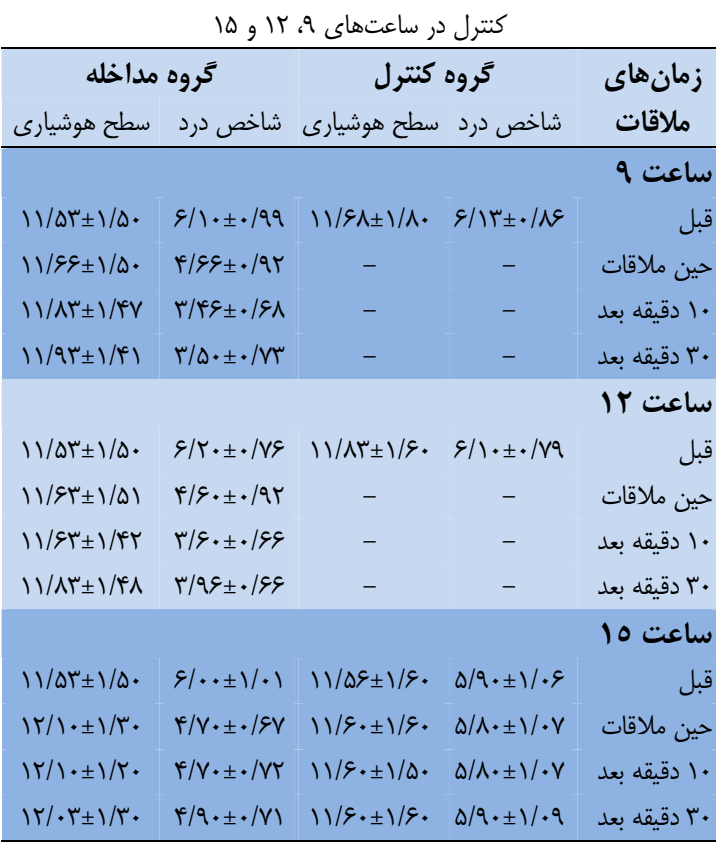

در كروه مداخله بين شاخصهاى درد اندازهخيرىشده در ساعتهاى

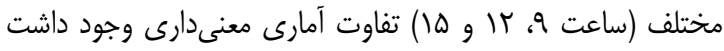

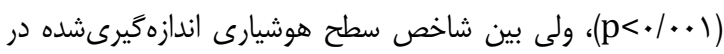

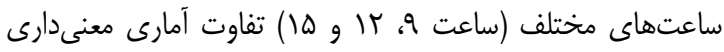

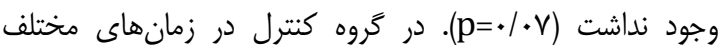

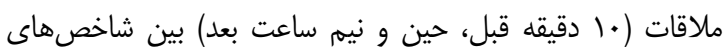

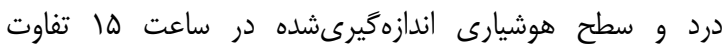

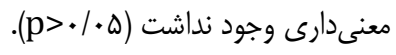

\section{بحث}

نتايج نشان داد قبل از انجام مداخله در دو كروه مداخله و كنترل

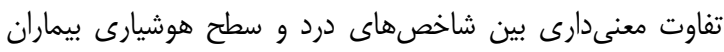

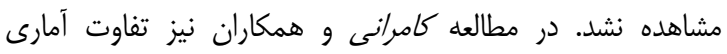

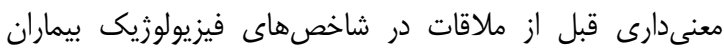
وجود نداشت كه اين يافته همكنبودن گروهها را ا اثبات مى كند [18].

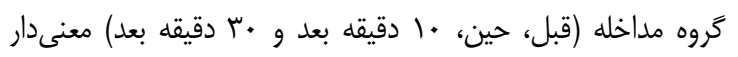

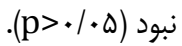

جدول () توزيع فراوانى واحدهاى مورد برؤهش (اعداد داخل برانتز ٪ هستند)

\begin{tabular}{|c|c|c|c|}
\hline معنى دارى & 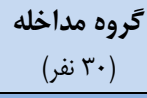 & 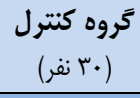 & متغير ها \\
\hline$\cdot / \mathrm{V}$ & 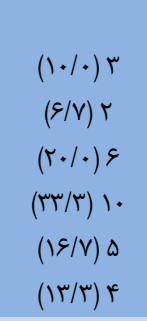 & 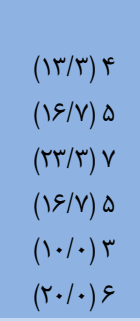 & 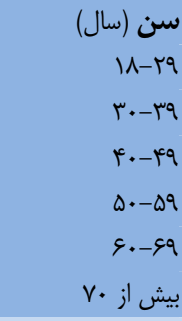 \\
\hline $1 /$. & 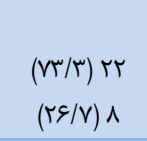 & $\begin{array}{l}(V / T / T) r T \\
(r / V) \wedge\end{array}$ & مجاهل \\
\hline.$/ 11$ & 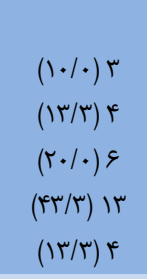 & $\begin{array}{l}(r / r) \backslash \\
(1 \cdot / \cdot)^{r} r \\
(r \cdot / \cdot)^{Y} \\
(\Delta S / V) \backslash V \\
(1 \cdot / \cdot)^{r}\end{array}$ & 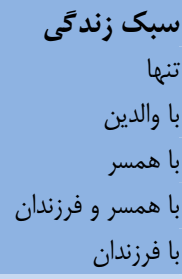 \\
\hline.$|8|$ & $\begin{array}{l}(g r / \%) 19 \\
(\Psi / V) 11\end{array}$ & $\begin{array}{l}(\Delta S / V) \text { IV } \\
\text { זו }\end{array}$ & زرد \\
\hline.$/ 99$ & $\begin{array}{l}(r g / V) I^{r} \\
(I T / r) r^{r} \\
(r \cdot / \cdot)^{q} \\
(I r / T)^{r} \\
(g / V)^{r}\end{array}$ & 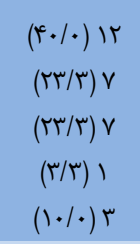 & ديسته مسوم مغزى \\
\hline . & $\begin{array}{l}(1 \cdot / \cdot)_{r}^{r} \\
(r / r / r) 1 . \\
(I r / r) r \\
(\varepsilon / V) r \\
(\varepsilon / V) r \\
(r \cdot / \cdot)^{q} q\end{array}$ & $\begin{array}{l}(r \cdot \%)^{q} \\
(r / r) v \\
(r . / \%) q \\
(\varphi / v) r \\
(1 . \%) r \\
(1 . \%) r\end{array}$ & 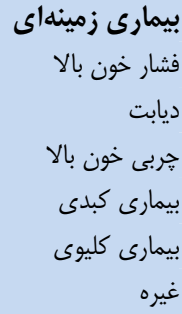 \\
\hline
\end{tabular}

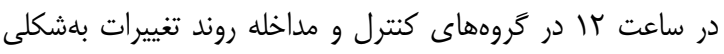
بود كه شاخص درد حين ملاقات كاهش معنى دارى نسبت به به قبل كاتل

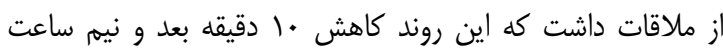

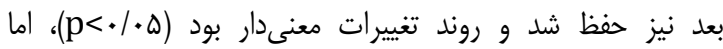

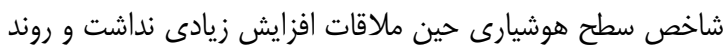

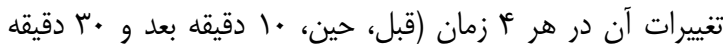

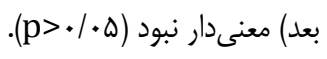


تاثير افزايش زمان ملاقات بر شاخصهاى درد و سطح هوشيارى بيماران بسترى در بخش مراقبتهاى ويزه 99

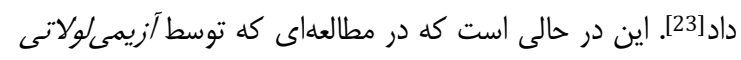

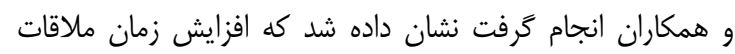

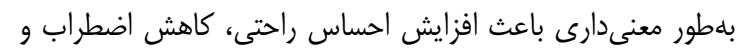

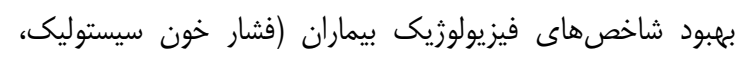

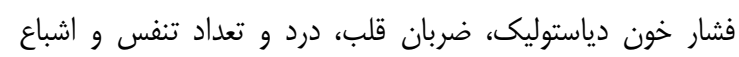

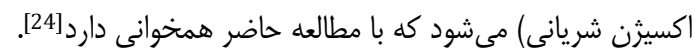

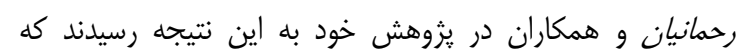

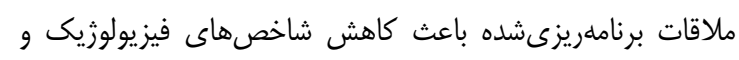

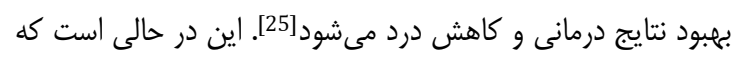

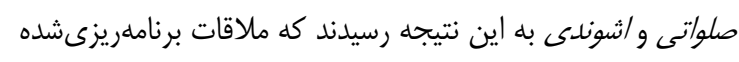

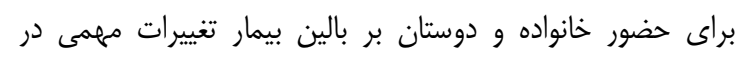

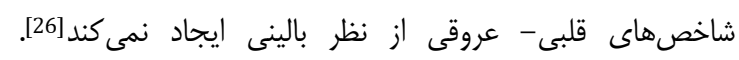

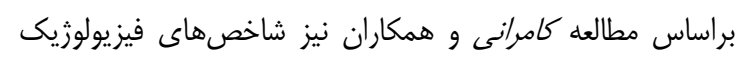
بيماران طى ملاقات افزايش يِيدا مى كند [18].

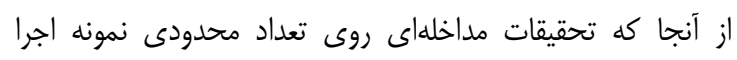

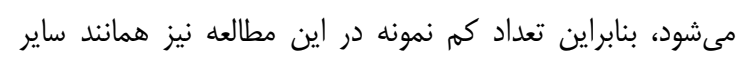

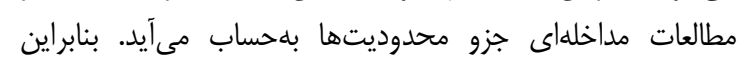

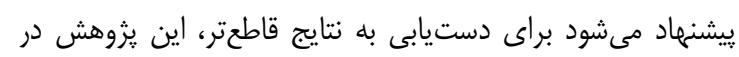

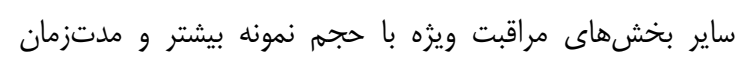

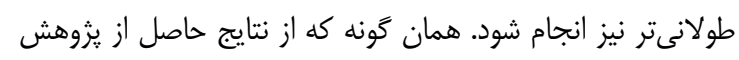

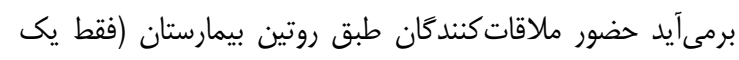

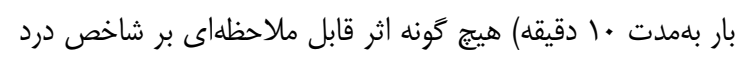

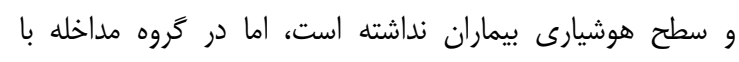

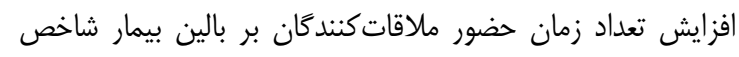

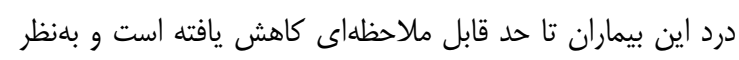

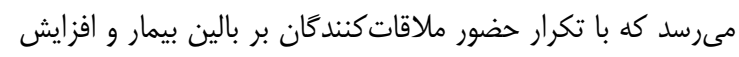

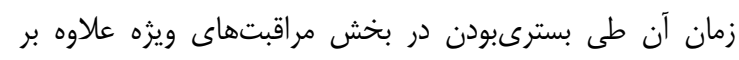

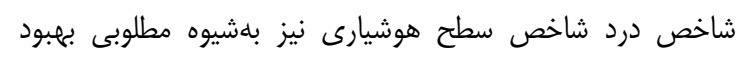

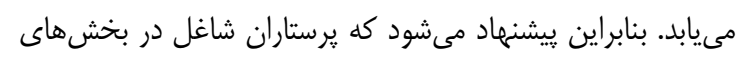

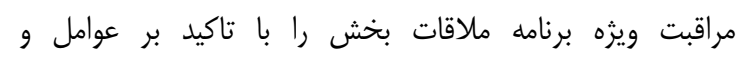

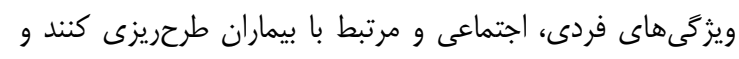

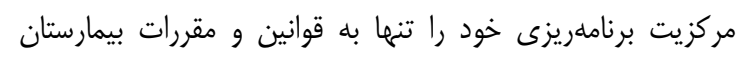

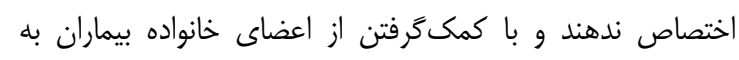

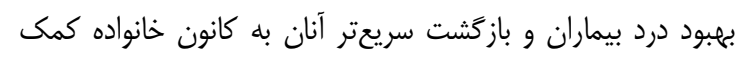

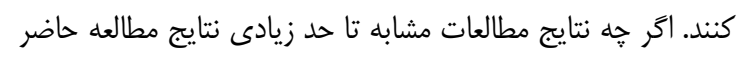

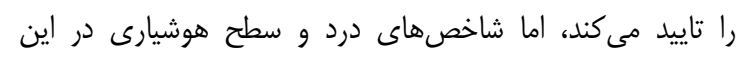

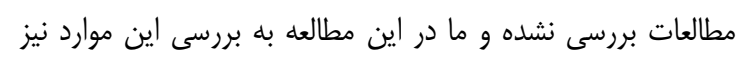

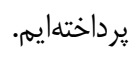

\section{نتيجه كَيرى}

افزايش زمان ملاقات باعث كاهش ميانكَين شاخص درد در بيماران بسترى در بخش آى سلى مانو مى باعثد.
نتايج مطالعه نشان داد در مرحله اول ملاقات (ساعت 9 صبح)، دوم

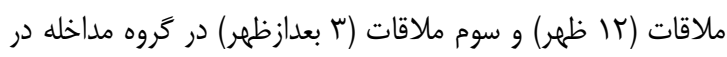

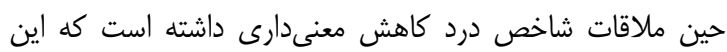

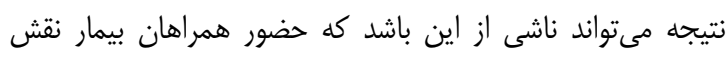

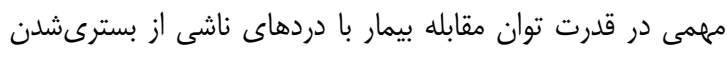

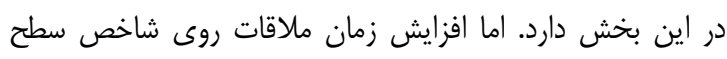

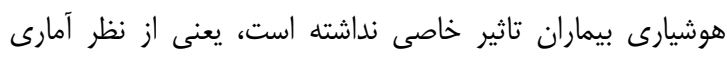

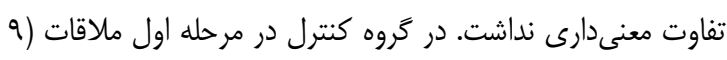

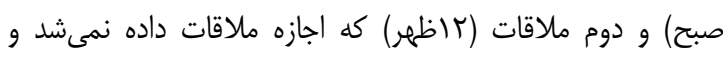

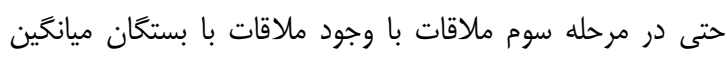
كليه شاخصها از نظر آمارى تفاوت معنى دارى ندات نداشت.

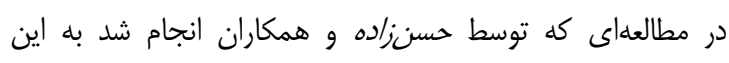

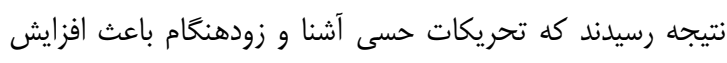

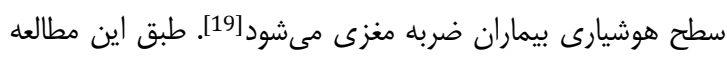

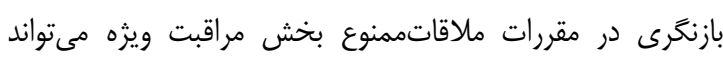

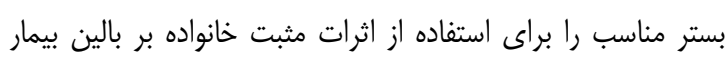

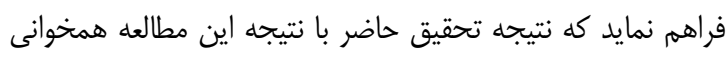

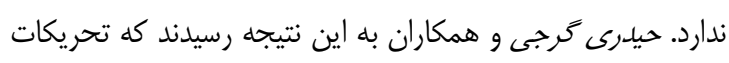

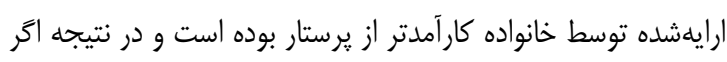

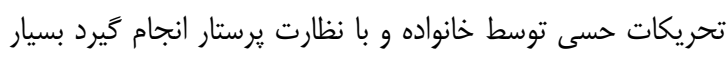

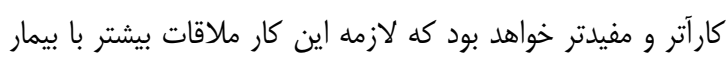

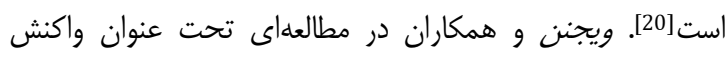

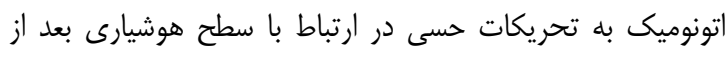

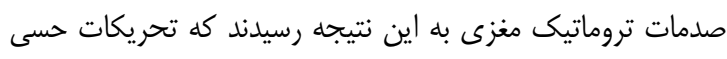

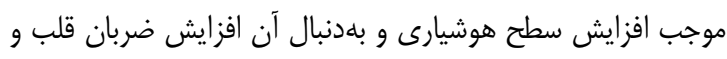

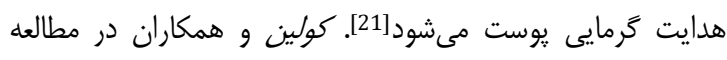

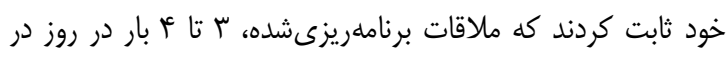

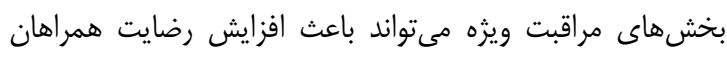

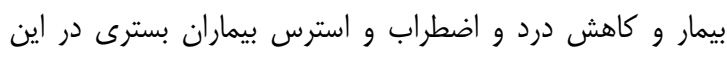

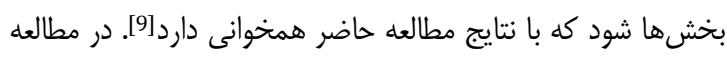

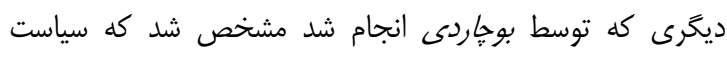

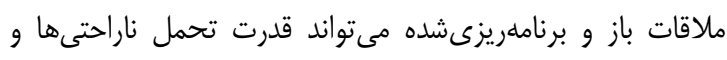

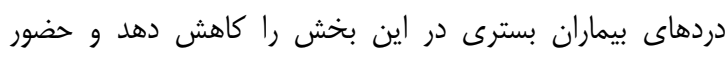

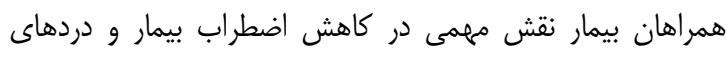

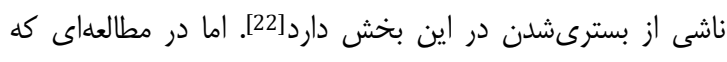

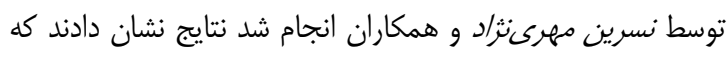

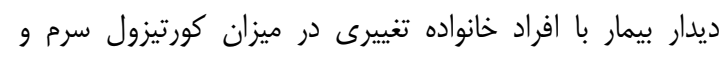
شاخصهاى فيزيولوزيك (فشار خون سيستوليى و دياستوليك)

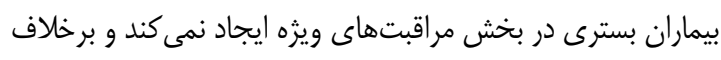

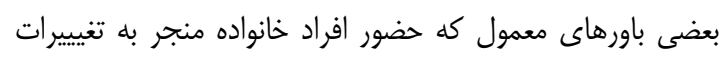

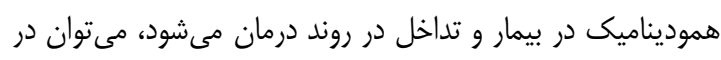

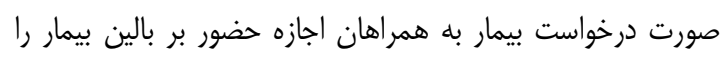


of ischemic heart disease epidemiology. Payesh. 2009;8(2):163-70. [Persian]

13- Goudarzi F, Basampoor S, Zakeri-Moghadam M, Faghih-Zadeh S, Rezaie F, Mohamad-Zadeh F. Changes in level of consciousness during auditory stimulation by familiar voice in comatosepatients. Iran J Nurs. 2010;23(63):43-50. [Persian].

14- Wiess SJ. Effects of differential touch on nervous system arousal of patients recovering from cardiac disease. Heart Lung. 1990;19(5pt1):474-80.

15- Nasiri M, Rahimiyan B, Jahanshahi M, Hajiyan K, Nikfar J. Stressors associated with hospitalization in the stressful cardiac care unit. Iran J Crit Care Nurs. 2011;4(3):141-8.

16- Ozer ZC, Senuzun F, Tokem Y. Evaluation of anxiety and depression levels in patients with myocardial infarction. Turk Kardiyoloji Dernegi Arsivi. 2009;37(8):557-62. [Turkish]

17- Gay EB, Pronovost PJ, Bassett RD, Nelson JE. The intensive care unit family meeting: Making it happen. J Crit Care. 2012;24(4):629e1-12.

18- Kamrani F, Seyed Javadi M, Abed Saiidi G, Ezati J Alavi Majd H, Hoseinian E. Comparison of physiological parameters of patients before, during and after the meeting in the Imam Khomeini hospital cardiac intensive care Ardebil. J Fac Nurs Midwifery Shahid Beheshti Univ Med Sci. 2010;20:18-22. [Persian]

19- Hasanzadeh F, Hoseini Azizi T, Esmaily H, Ehsaee MR. The impact of familiar sensory stimulation on level of consciousness in patients with head injury in ICU. J North Khorasan Univ Med Sci. 2012;4(1):121-9. [Persian]

20- Heydari Gorji MA, Araghian Mojarad F, Jafari H, Gholipour A, Yazdani Cherati J. Comparing the effects of familiar and unfamiliar voices as auditory sensory stimulation in level of consciousness among traumatic comatose patients in intensive care unit. J Mazandaran Univ Med Sci. 2013;22(97):208-14. [Persian]

21- Wijnen VJ, Hevtink M, van Boxtel GJ, Eilander HJ, de Gelder B. Autonomic reactivity to sensory stimulation is related to consciousness level after severe traumatic brain injury. Clin Neurophysiol. 2006;117(8):1794-807.

22- Burchardi H. Let's open the door. Intensive Care Med. 2002;28(10):1371-2.

23- Mehrnejad N, Navid Hamidi M, Rezaei Homami M, Ganji T, Ardeshiri M, Fathi P. The effect of family presence at the bedside on serum cortisol levels and physiological indexes in patients hospitalized in intensive care unit. Cardiovasc Nurs J. 2014;2(4):36-48. [Persian]

24- Azimi Lolaty H, Bagheri-Nezami M, Shorofi SA Golzaridi T, Yazdani charati J. The effects of family-friend visits on anxiety, physiological indices and well-being of MI patients admitted to a coronary care unit. Complement Ther Clinical Pract. 2014;20(3):147-51.

25- Rahmani R, Ahmadian Yazdi R, Motahedian Tabrizi E, Rahimi A. To assess the effect of planed meeting on the physiologic indicators of the patients who suffer from acute coronary syndrome. Iran J Crit Care Nurs. 2013;6(1):57-64. [Persian]

26- Salavati M, Najafvanzade M, Oshvandi K, Homayoonfar S, Soltanian A. Effect of scheduled appointments on physiological parametr in the Intensive cardiac patients unit. Hamedan J Fac Nurs Midwifery. 2012;44:41-51. [Persian]

$$
\begin{aligned}
& \text { تشكر و قدردانى: بدين وسيله از اعضاى محترم شوراى }
\end{aligned}
$$

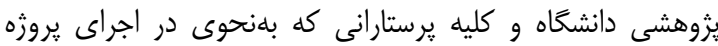

$$
\begin{aligned}
& \text { مشاركت داشتند تشكر بdعمل مئي دآيد. }
\end{aligned}
$$

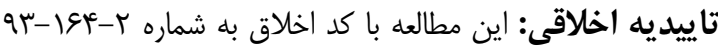

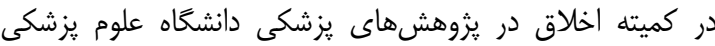

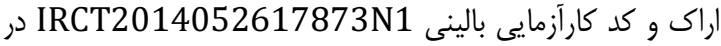

$$
\begin{aligned}
& \text { مركز ثبت كارآزمايى بالينى ايران به ثبت رسيده است. }
\end{aligned}
$$

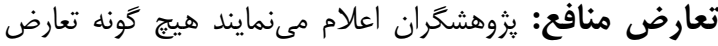

$$
\begin{aligned}
& \text { منافع در اين يثروهش و مقاله حاصل از آن وجود ندارد. مئرون } \\
& \text { منابع مالى: كليه منابع مالى اين طرح تحقيقاتى (با شماره II OVV) }
\end{aligned}
$$

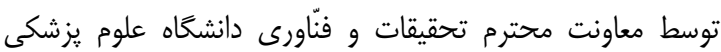

$$
\begin{aligned}
& \text { اراك تامين شده است. }
\end{aligned}
$$

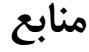

1- Simini B. Patients' perceptions of intensive care. Lancet. 1999;354(9187):571-2.

2- Berti D, Ferdinande P, Moons P. Beliefs and attitudes of intensive care nurses toward visits and open visiting policy. Intensive Care Med. 2007;33(6):1060-5.

3- Davidson JE, Powers K, Hedayat KM, Tieszen M, Kon AA, Shepard E, et al. Clinical practice guidelines for support of the family in the patient-centered intensive care unit: American College of Critical Care Medicine Task Force 2004-2005. Crit Care Med. 2005;35(2):60522.

4- Biancofiore G, Bindi ML, Romanelli AM, Urbani L, Mosca F, Filipponi F. Stress-inducing factors in ICUs: What liver transplan recipients experience and what caregivers perceive. Liver Transplant, 2005;11(8):96772.

5- Simon SK, Phillips K, Badalamenti S, Ohlert J, Krumberger J. Current practices regarding visitation policies in critical care units. Am J Crit Care. 1997;6(3):210-7.

6- Berwick D, Kotagal M. Restricted visiting hours in ICUs: Time to change. JAMA. 2004;292(6):736-7.

7- Nelson JE, Meier DE, Oei EJ, Nierman DM, Senzel RS, Manfredi PL, et al. Self-reported symptom experience of critically ill cancer patients receiving intensive care. Crit Care Med. 2001;29(2):277-82.

8- Spreen AE, Schuurmans MJ. Visiting policies in the adult intensive care units: a complete survey of Dutch ICUs. Intensive Crit Care Nurs. 2011;27(1):27-30.

9- Gonzalez CE, Carroll DL, Elliott JS, Fitzgerald PA, Vallent HJ. Visiting preferences of patients in the intensive care unit and in a complex care medical unit. Am J Crit Care. 2004;13(3):194-8.

10- Haghbin S, Tayebi Z, Abbasian A, Haghbin H. Visiting hour policies in intensive care units, southern Iran. Iran Red Crescent Med J. 2011:13(9):684-686

11- Linsfumis RR, Ranzani0, FariaPP, Schettino G. Anxiety, depression, and satisfaction in close relatives of patients in an open visiting policy intensive care unit in Brazil. J Crit Care. 2015;30(2):440.e1-6.

12- Talebizadeh N, Haghdoost AA, Mirzazadeh A. Models 